\title{
Saber pedagógico: fundamento del ejercicio docente ${ }^{1}$
}

\section{Tomás Sánchez-Amaya}

Universidad Distrital Francisco José de Caldas, Colombia

tsancheza@udistrital.edu.co

\section{Hamlet Santiago González-Melo}

Universidad Distrital Francisco José de Caldas, Colombia hsgonzalezm@udistrital.edu.co

\section{Resumen}

El presente artículo de reflexión comporta un ejercicio analitico descriptivo e interpretativo, que indaga por la vigencia, importancia y necesidad de resignificar el saber pedagógico del docente, en tanto saber fundamental de su práctica. En el análisis, se realizaron unas consideraciones iniciales en torno al saber en general y la caracterización del saber pedagógico en particular, con el objeto de ubicar la analitica en un campo especifico: la arqueología, desde una perspectiva foucaultiana. A continuación, se abordaron de manera sucinta algunas relaciones que son connaturales entre el saber pedagógico y el poder (en tanto manifestación de las relaciones humanas), que se traman en la acción pedagógica. Se realizó, posteriormente, un breve inventario de los elementos que configuran el saber pedagógico; el ejercicio finaliza con el cuestionamiento de si es el maestro el sujeto depositario, poseedor, constructor y reconstructor de dicho saber.

\section{Palabras clave}

Teoría de la educación; saber educativo; saber pedagógico; práctica pedagógica; formación de profesores (Fuente: Tesauro de la Unesco).

1 Este ejercicio se adscribe a la propuesta de investigación interinstitucional e internacional "Analítica descriptiva e interpretativa de la formación docente. Los casos de las universidades: Distrital Francisco José de Caldas (Bogotá, Colombia), Nacional de San Luis (San Luis, Argentina) y Universidad del Tolima (Ibagué, Colombia)", aprobada por la Universidad Distrital Francisco José de Caldas a través de la Convocatoria o 8 de 2014 del Centro de Investigaciones y Desarrollo Científico (Acta 12-2014). La propuesta se fundamenta teórica y metodológicamente en los libros La evaluación educativa en Colombia: segunda mitad del siglo XX. Esbozo de una genealogía (Sánchez, 2015) y Caracterización del saber pedagógico de los profesores del Proyecto Académico de Investigación y Extensión de Pedagogía-PAIEP en la Universidad Distrital Francisco José de Caldas (González, 2016).

Recepción: 2/10/2015 | Envío a pares: 15/10/2015 | Aceptación por pares: 16/o2/2016 | Aprobación: 16/04/2016 


\title{
Pedagogical Know-how: The Basis of Teaching Practice
}

\begin{abstract}
This critique involves a descriptive and interpretative analytical exercise that explores the validity, importance and necessity of redefining the teacher's pedagogical know-how, inasmuch as it is fundamental knowledge for teaching practice. The analysis involves initial considerations about knowledge in general and the characterization of pedagogical know-how in particular, the idea being to situate the analysis in a specific field; namely, that of Foucault's archeology. Several relationships are addressed succinctly, specifically ones that are connatural between pedagogical know-how and power (as a manifestation of human relations), and are woven into the act of teaching. A brief inventory of the elements that make up pedagogical know-how is provided as well. The exercise ends by asking if it is the teacher who is the master custodian, possessor, builder and rebuilder of that know-how.
\end{abstract}

\section{Keywords}

Education theory, educational knowledge, pedagogical know-how, teaching practice; teacher training (Source: Unesco Thesaurus). 


\title{
Saber pedagógico: fundamento do exercício docente
}

\author{
Resumo
}

Este artigo de reflexão compreende um exercício analítico descritivo e interpretativo que questiona sobre a validade, a importância e a necessidade de ressignificar o saber pedagógico do docente enquanto saber fundamental de sua prática. Na análise, foram realizadas considerações iniciais sobre o saber em geral e a caracterização de saber pedagógico em particular, com o objetivo de posicionar a analítica num campo especifico: a arqueologia, sob uma perspectiva foucaultiana. A seguir, foram abordadas, de maneira sucinta, algumas relações que são conaturais entre o saber pedagógico e o poder (enquanto manifestação das relações humanas), que são estabelecidas na ação pedagógica. Em seguida, realizou-se um breve inventário dos elementos que configuram o saber pedagógico; o exercício finaliza com o questionamento de se é o mestre o sujeito depositário, possuidor, construtor e reconstrutor desse saber.

\section{Palavras-chave}

Teoria da educação, saber educativo, saber pedagógico, prática pedagógica, formação de professores (Fonte: Tesauro da Unesco). 


\section{Introducción}

El presente artículo de reflexión se refiere al saber pedagógico en tanto fundamento del ejercicio docente; saber que implica diferentes relaciones inter y transdisciplinares que son puestas en juego en la acción cotidiana docente, en el aula de clase y, en general, en la labor educativa. El propósito se ordena a abordar algunas comprensiones sobre el saber (pedagógico) y sus relaciones con el poder; asimismo, por la condición del docente -en tanto sujeto depositario y productor de dicho saber-, y, por los modos en que, en los procesos educativoformativos, es puesto en funcionamiento. Se busca explorar, en consecuencia, las formas como el saber pedagógico se constituye en condición de posibilidad para la creación de didácticas, ${ }^{2}$ de mediaciones, de herramientas, de métodos y de estrategias en el aula y en otros escenarios educativos en los que discurre el ejercicio docente.

Como fundamentos de orden metodológico en los que se apoyan los análisis, se plantean, en primera instancia, algunos desarrollos teóricos de Foucault (2006, 2004), específicamente los referidos a la denominada caja de herramientas (perspectivas arqueológica y genealógica) en tanto dispositivos de análisis de saberes - marginales y marginados de la gran teoría-, saberes de los bordes, subyacentes a las propias prácticas sociales (Sánchez, 2008). A este respecto, coincidimos también con Echeverry (1998) en lo referido a la importancia de definir de conceptos de frontera que permitan captar la diversidad y los intentos de formalización que se han hecho de la pedagogía y de la didáctica desde las ciencias naturales y sociales.

2 Compartimos concepciones con Vasco (1990) y De Tezanos (2007); quienes señalan a la didáctica como aquella disciplina que tiene por objeto de estudio la enseñanza, aunque tal disciplina no dé cuenta de la totalidad del ejercicio de enseñar. Aclaramos, no obstante, que es necesario tener cuidado con los riesgos que pueden correrse si se postula como aspecto principal en la formación de maestros, así como su carácter reduccionista en términos de las posibilidades que puede implicar su uso acrítico.
La propuesta foucaultiana es abordada - y en cierta medida puesta en funcionamiento- por el Grupo de la Historia de las Prácticas Pedagógicas en Colombia, grupo interdisciplinar y heterogéneo - como el saber pedagógico mismo- que, concibiendo la pedagogía como saber, abre innumerables espacios para la construcción de historias particulares acerca de sus dominios relativos y correlativos. Este conjunto de desarrollos teóricos permite, de diversos modos, cartografiar, describir, mapear, territorializar, configurar y reconfigurar el saber pedagógico, sus concepciones, sus formas, sus relaciones, sus fuerzas, sus objetos y, por qué no, los sujetos.

El plan de obra trazado para tratar de dar cuenta de la propuesta contempla los siguientes apartados: inicialmente, se propone una reflexión sobre algunas consideraciones en torno al saber pedagógico, ello implica un acercamiento conceptual a las determinaciones teóricas que permiten sus posibilidades de enunciación; a continuación, se realiza una aproximación a las relaciones entre saber y poder, que hacen posible la emergencia del saber pedagógico; posteriormente, se abordan los elementos presentes en la construcción del saber pedagógico; y, finalmente, se explorarán algunos sentidos del docente como sujeto depositario del saber pedagógico; aquí también se analizarán las posibilidades que dicho saber aporta a la transformación de la práctica pedagógica.

\section{Consideraciones iniciales en torno al saber pedagógico}

Antes de abordar lo relativo al saber pedagógico, convendría poner de relieve lo que entendemos por saber, para luego detenernos en el análisis del saber con su determinante "pedagógico". En otras palabras, ¿qué es lo pedagógico del saber pedagógico?

Para abordar dicho cuestionamiento, es necesario referirse a una pregunta anterior: ¿qué es un saber, o mejor, qué es el saber? Los referentes teóricos mencionados arriba nos aportan diversas fun- 
damentaciones. Al respecto, Foucault (2006) concibe el saber como: todo aquello que se puede decir, que se puede nombrar, que se puede enunciar de un objeto (tema, problema, estrategia...) en una práctica discursiva $^{3}$ determinada; el dominio de los objetos que tienen -o no- pretensiones de cientificidad; el campo de correlación o de subordinación de los enunciados en los cuales los objetos aparecen, emergen, se transforman, forman dispersiones, traman una red de relaciones y regularidades que constituyen las condiciones de posibilidad de la emergencia de los discursos.

Al decir de González y Florián (1999),

...los avances epistemológicos se suceden a partir de las rupturas con relación al pensamiento tradicional. Esta forma de pensar el conocimiento no solo aumenta nuestras posibilidades de expresión sobre lo que observamos, sino que también nos pone en estado de alerta en cuanto a muchos prejuicios y acontecimientos tomados como ciertos o indiscutibles en la conformación de la historia de Occidente, y de la verdad constituida socialmente (p.95)

Aquí es clara la crítica que se realiza a las concepciones metafísicas, totalitarias, absolutas y dogmáticas que pueden avocar ciertos abordajes tradicionales para leer la realidad.

De igual manera, Foucault (2004) se refiere a diferentes formas como es posible enfocar diversos análisis en relación con los objetos que se examinan, en función de obtener una perspectiva de abordaje más profunda, diferente y diferencial:

3 Entendemos, siguiendo los desarrollos teóricos de Foucault, por prácticas discursivas, al "conjunto de reglas anónimas, históricas, siempre determinadas en el tiempo y en el espacio que han definido en una época dada, y para un área social, económica, geográfica o lingüística dada, las condiciones de ejercicio de la función enunciativa" (2006, p. 198). Las prácticas discursivas comportan, en consecuencia, una materialidad específica: se refieren a un hacer (práctica) y a un saber (discursos) a la manera de eslabones de una misma cadena, o sucesiones teórico-prácticas que se explicitan en las mismas prácticas sociales.
Este pensamiento que se mantiene fuera de toda subjetividad para hacer surgir como del exterior sus límites, enunciar su fin, hacer brillar su dispersión y no obtener más que su irrefutable ausencia, y que al mismo tiempo se mantiene en el umbral de toda positividad, no tanto para extraer su fundamento o su justificación, cuanto para encontrar el espacio en que se despliega, el vacío que le sirve de lugar, la distancia en que se constituye y en la que se esfuman, desde el momento en que es objeto de la mirada, sus certidumbres inmediatas -este pensamiento, con relación a la interioridad de nuestra reflexión filosófica y con relación a la positividad de nuestro saber, constituye lo que podemos llamar el pensamiento del afuera (pp.16-17).

En relación con el conocimiento, afirma que este "abate las protecciones ilusorias; deshace la unidad del sujeto, libera en él todo lo que se empeña en disociarlo y destruirlo" (Foucault, 2000, p. 71).4

De igual manera, asocia la misma génesis de las prácticas sociales al saber en la medida que estas (prácticas jurídicas o judiciales), engendran nuevos dominios de saber, nuevos sujetos de conocimiento, los cuales propenden por la emergencia de nuevas formas de subjetividad. Por ejemplo, en el siglo XIX se forma un saber del hombre, de la individualidad, del individuo normal o anormal, dentro o fuera de la regla. Es importante mencionar que este saber surgió de las prácticas sociales de control y vigilancia; no fue un saber impuesto (González y Florián, 1999).

Por su parte, Zuluaga (1987) muestra el saber como el conjunto de conocimientos de los más diversos niveles, unos de los cuales apenas se dejan esbozar a través de objetos o de modalidades de enunciación y que no han logrado aún estatuto de formalización; otros saberes que, habiendo franqueado determinados umbrales (de epistemologización), se perfilan en la perspectiva de lograr

$4 \quad$ En este punto está muy presente la influencia de Nietzsche y su crítica al carácter absoluto y prescriptivo de la verdad. 
determinado estatuto de cientificidad: saber que ha podido franquear el umbral de la formalización, saber que es reconocido y puesto en funcionamiento por una comunidad específica.

En cuanto al saber pedagógico, este configura discursos sobre la educación y la pedagogía que poseen una identidad y obedecen a ciertas reglas. Dichos discursos establecen tendencias, modos de ver y de actuar, en relación con las determinaciones de las que hacen parte. Este saber que emerge de unas prácticas sociales, en algunos casos también institucionalizadas, a la vez, es modelado en los discursos que lo constituyen a partir de las delimitaciones que lo configuran. Su potencia radica en los alcances metodológicos, estratégicos y procedimentales que plantean, en cuanto sirve para:

a) [...] reunir discursos a propósito de la enseñanza y la educación; es por tanto un conjunto pero de elementos dispares. No reúne para conformar un cuerpo conceptual sino para reconstruir un conjunto asi definido por unas reglas que le confieren unidad [...] En suma, constituye la reconstrucción mediante un proceso de investigación, y en este sentido las posibilidades de construcción son numerosas.

b) Así mismo, el saber pedagógico constituye la condición de existencia, al interior de una práctica especifica, de proposiciones coherentes, descripciones más o menos exactas, teorías, análisis cuantitativos y normas, formando un campo heterogéneo con los discursos correspondientes a este conjunto. Por esto, no existe saber sin una práctica definida y toda práctica se perfila por el saber que forma. En consecuencia, un saber no podría constituirse sin una práctica que le confiriese materialidad: es la manera como los conocimientos entran en acción en una sociedad.

c) Todo saber está conformado por elementos que lo hacen identificable en su propio mo- mento histórico y posteriormente asequible y reconstruible al trabajo arqueológico.

d) Los diferentes objetos en torno a los cuales giran los discursos y las prácticas [...].

e) Los conceptos que aparecen, se definen, se aplican y se transforman y que pertenecen a los conocimientos agrupados en la práctica [...].

g) Las posibilidades de utilización y apropiación dispuestas por el discurso mismo o por las regulaciones externas a la práctica (Zuluaga et al., 2006, pp. 94-95).

Si bien estas consideraciones, que pueden y deben ser objeto de ulteriores análisis, delimitación conceptual y contrastes con sus correlatos prácticos, propician formas novedosas de pensar la génesis de muchas de las conceptualizaciones que continuamente realizan los maestros en su quehacer cotidiano.

\section{El saber constituido en las relaciones de poder}

El saber, señala Zuluaga (1987), se constituye en espacios de construcción social, emergentes de recortes de saberes disciplinares, de ciencias o de disciplinas que tengan alguna relación con la práctica discursiva de la que efectivamente algo se dice o se enuncia. Igualmente, constituye instancias de regulación, de marcación y demarcación de los discursos, de los sujetos y de los poderes, al modo que se constituyen en condición de posibilidad, también, para la estructuración de las relaciones de poder y de la configuración de sujetos; aquellos saberes son, a la vez, configurados y reconfigurados por dichos sujetos.

De esta manera, las sociedades determinan los discursos y las relaciones de poder que han de ser puestas en funcionamiento y a la vez son determinadas por estos (Foucault, 2003).

La relación poder-saber está plenamente identificada en Foucault, en cuanto las formas de conocimiento están articuladas al poder imperante: "No 
hay en el conocimiento una adecuación al objeto, una asimilación sino que hay, por el contrario, una relación de distancia y dominación [...] El carácter perspectivo del conocimiento no deriva de la naturaleza humana sino siempre del carácter polémico y estratégico del conocimiento" (2003, p. 30). Al respecto, el pensador francés se refiere a la formación desde diversas posibilidades que emergen y plantean nuevos retos y abordajes en el análisis de lo social, "ciertos tipos de sujeto de conocimiento, órdenes de verdad, dominios de saber, a partir de condiciones políticas, que son como el suelo en que se forman el sujeto, los dominios de saber y las relaciones de verdad" (p. 32). Así pues, el poder permite el establecimiento de varios tipos de saberes. Tal es el caso de la postulación de las llamadas ciencias humanas y del hombre como objeto de estudio científico. En consecuencia, el poder genera discursos, saberes, conductas, técnicas, producciones que son estudiadas por Foucault (Cf. González y Florián, 1990). ${ }^{5}$

Quizá convenga un ejemplo para tratar de concretar lo enunciado: siguiendo los lineamientos de Foucault en sus arqueologías y genealogías (el saber clínico, la locura, la prisión, las sociedades disciplinarias, la sexualidad), al preguntarse, por ejemplo, por los elementos, sujetos e instancias que convergen en la configuración de un saber determinado (la locura), encuentra que existe una enorme dispersión de enunciados o de formas enunciativas a través de los cuales se organiza, teniendo en consideración unas condiciones determinadas, un saber, el saber acerca de la locura. Fragmentos de diversos discursos disciplinarios y científicos aportan a la configuración de ese saber (la medicina, la psiquiatría, el derecho, la jurisprudencia, la administración, la economía, la biología, la moral, etc.); diversos sujetos hablan y saben de locura (los locos, los vigilantes, los médicos, los abogados, los curas, la familia de los internos...);

5 La construcción de dichos estudios e indagaciones plantea una gran novedad, en lo que se había constituido como objeto de la tradición filosófica, a excepción de Spinoza y Nietzsche, quienes también se habían aproximado a lo humano desde sus propias perspectivas. diversas instituciones se arrogan el saber sobre la locura (el manicomio, el hospital, etc.). La composición, el conjunto de todo ese complejo de fragmentos de saberes constituye, al decir del autor, el saber sobre la locura.

Una vez conceptualizado el saber como todo aquello que se puede enunciar de una práctica discursiva determinada, podemos decir: el saber pedagógico sería todo aquello que se puede decir, que se puede enunciar, acerca de la pedagogía o de las prácticas pedagógicas, que un sujeto -el maestro- pone en funcionamiento en el acto de enseñar, esto es, el fundamento de su práctica.

Concretemos mediante un ejemplo, parodiando lo referido al saber sobre la locura, lo relativo al saber pedagógico; preguntemos, por ejemplo, ¿qué sujetos pueden enunciar algo acerca de la pedagogía, de la educación, de la instrucción, de la formación, del currículo, de la evaluación, de los planes de estudio, de los programas y procesos, de las acciones propias de la acción de enseñar?; ¿qué disciplinas, ciencias o campos disciplinares erigen discursos relativos a este conjunto de elementos concernientes a la educación y a la pedagogía?; ¿en qué instituciones se formulan estos discursos?; ¿qué posiciones ocupan los sujetos que abordan los discursos acerca de la pedagogía?, ¿qué prácticas se ponen en funcionamiento en los procedimientos de enseñanza?; ¿qué relaciones se traman entre los sujetos, los poderes y los saberes relativos a las prácticas discursivas de la pedagogía?; en últimas, ¿qué es el saber pedagógico? Este conjunto de preguntas y, por supuesto, la multiplicidad y variedad de respuestas, constituirían el saber pedagógico.

\section{Elementos que interactúan en la construcción del saber pedagógico}

Avancemos un poco más en la concreción del saber pedagógico a partir de los elementos que lo constituyen y las determinaciones que se ciernen a su interior en procura de establecer su naturaleza y 
composición estructural. A continuación se postulan dichos componentes.

- Sujetos que enuncian discursos y prácticas relativas a la pedagogía: el maestro, el estudiante, los directivos, los administrativos, los técnicos y tecnócratas de la educación, los gobernantes (en todos los órdenes de la administración pública, desde lo general, nacional, hasta lo particular, municipal), los miembros de la familia y sus respectivos roles a la luz de los roles constituidos social y culturalmente, etc. En este caso puede identificarse, así mismo, la posición que cada uno de estos sujetos desempeña en relación con el discurso y con las relaciones de poder que los produce.

- Disciplinas, ciencias o campos disciplinares que erigen discursos relativos a la pedagogía: las ciencias de la educación, la misma pedagogía, la psicología, la sociología, las teorías acerca de los modelos curriculares, las teorías de la didáctica.

- La epistemología, las metodologías, las teorías acerca de la naturaleza, sus modelos y enfoques acerca de la educación, la pedagogía, la didáctica, la evaluación; los discursos de la planeación, el derecho, la política, la administración, la biología, las ciencias económicas, la filosofía, la ética, los discursos interdisciplinares modernos (neurobiología, psicobiología, sociogenética, bioética, etc.), los saberes de sentido común, saberes otros, saberes de la vida, saberes experienciales, cotidianos y emergentes, y todos aquellos de la experiencia propia de los sujetos implicados en la acción pedagógica. ${ }^{6}$

$6 \quad$ Estos saberes experienciales-vivenciales de la cotidianidad son recurrentemente referenciados en diferentes estudios, investigaciones y abordajes en relación con la génesis del saber pedagógico. Para mayor ilustración sugerimos revisar la Tesis Doctoral —en curso-Caracterización del saber pedagógico de los profesores del Proyecto Académico de Investigación y Extensión de Pedagogía-PAIEP de la Universidad Distrital Francisco José de Caldas (González, 2016).
- Instituciones en las que se formulan estos discursos: la escuela (en todas sus formas, manifestaciones y niveles), las instancias administrativas, las instancias docentes, los escenarios donde se elaboran leyes y discursos prescriptivos, las instituciones disciplinarias (la familia, el taller, el cuartel, la fábrica, las escuela, etc.).

- El conjunto de prácticas que los sujetos, las instituciones y las diferentes instancias ponen en funcionamiento en los procedimientos de enseñanza y sus formas de control asociadas a la generación de un cierto tipo de sujeto de acuerdo con circunstancias, así como las determinantes sociohistóricas en la cuales se sitúan y emergen.

- El saber constituido en torno a los objetos de la educación y la pedagogía: modelos, teorías y enfoques de aprendizaje, de desarrollo de los sujetos, de los modelos pedagógicos, de los modelos evaluativos, métodos de enseñanza, de proyectos y procesos que intervienen en la acción pedagógica, etc.

- El conjunto de relaciones que se dan entre los sujetos de la pedagogía y los saberes que median tales relaciones.

Siguiendo los desarrollos teóricos de Foucault (1979), dicho acumulado constituye, a su vez, formas de poder y micropoderes, así como planteamientos de resistencia, que pueden proyectarse en un sentido de producción de nuevo saber y acciones, en consecuencia, con las prescripciones de aquel.

Todo este saber constituido de recortes y fragmentos de otros saberes afines, cercanos y complementarios a la pedagogía, constituye el saber pedagógico, el cual es propio del docente y se pone en funcionamiento en el ejercicio de enseñar. El saber pedagógico, entonces, es un saber constituido por un conjunto plural de saberes, de discursos, de determinaciones, de instituciones sociales al servicio de sus propios intereses. Dichos elementos plantean reflexiones en torno a las prácticas que versan sobre la pedagogía 
y otros territorios discursivos - $\mathrm{O}$ no discursivosfronterizos.

\section{El maestro: ¿sujeto depositario del saber pedagógico?}

El conjunto de saberes, de objetos, de elementos que hemos referido, tienen relación con la acción pedagógica; esta acción, al decir de Zuluaga (1987), Vasco (1990), Tamayo (2007), entre otros, es propia de un sujeto que desempeña el rol de maestro. El maestro es el sujeto primario y fundamental de la pedagogía, es quien acopia un saber que, como hemos visto, trasciende los límites de la pedagogía para anclarse en los discursos inter y transdisciplinarios que se plantean en la escena del ejercicio de la enseñanza.

Zuluaga (1987) sostiene que tras la instalación de las facultades de educación en Colombia, se legó a los maestros la función de enseñar, unos anclan su saber a métodos, teorías y modelos pedagógicos, de enseñanza y aprendizaje; otros desempeñan esa función en relación con saberes disciplinares de otros discursos separados o alejados de la pedagogía; esto facultará para decir que aunque todo pedagogo puede ser maestro, no todo maestro es pedagogo. Un ejemplo concreto de ello es lo que acontece con profesionales de diversas disciplinas que desempeñan roles docentes sin la suficiente fundamentación pedagógica, lo cual no quiere decir que la pedagogía se limite o se reduzca a la enseñanza de didácticas o metodologías para la acción de enseñar, como lo habíamos precisado más arriba.

Vasco (1990), en su artículo "El saber pedagógico: razón de ser de la pedagogía”, realizó una exposición en relación con dicho saber, allí señala que el saber pedagógico es el saber propio del maestro, el cual pone en funcionamiento en el acto cotidiano de enseñar. El maestro, señala, depositario de ese saber lo configura a partir de todo ese campo amplio de saberes y a partir de unas preguntas ineludibles a su propia función de enseñante y al mismo discur- so pedagógico: ¿qué enseñar?, ¿a quién se enseña?, ¿para qué se enseña?, ¿cómo enseñar?, ¿dónde y cuándo enseñar?

- $\quad$ En relación con la primera pregunta, ¿qué enseñar?, la respuesta inmediata podría ser: se enseña algo (una asignatura, unos contenidos, un área del conocimiento, unos procedimientos metodológicos para llegar a una finalidad, un curso determinado, una temática dada, unas problematizaciones específicas, etc.); cualquiera que sea la respuesta, sin embargo, se evidencia una relación entre unos sujetos (docente y discente) con un objeto de enseñanza determinado, un conocimiento dado. Esta relación denota una posición del maestro con el objeto propio de lo que enseña, con los desarrollos teóricos de la cátedra que orienta, con unos contenidos seleccionados, con unos temas específicos y unos modos concretos de cómo proceder en el proceso de enseñanza. En cualquier asignatura, de cualquier nivel educativo, el docente debe abordar unos contenidos seleccionados (por él mismo, por otros docentes, por la institución misma o por los entes reguladores y de control estatal).7

7 La relación del docente con el campo de conocimiento al que se adscribe la asignatura que orienta determina ciertos modos de concebir el conocimiento y la realidad que aquel describe. Por ejemplo, el fungir como docente en un departamento de formación humanística, orientando una cátedra de sistemas políticos que tenga un grupo base de docentes, exige que el profesor se acomode a los contenidos seleccionados por dicho equipo, a los objetivos, las temáticas y las problemáticas determinadas por ellos, y que responda a las exigencias y necesidades que la comunidad académica de dicha cátedra determine como fundamentales. Por supuesto, la selección de contenidos implicaría el dominio de unos saberes propios de la asignatura, lo cual consultará los desarrollos teóricos de la misma, dados a través de la historia y que a juicio del grupo son considerados los más importantes o que responden de mejor manera a las exigencias institucionales o sociales. Sobre esto pueden verse, también, los programas oficiales expedidos por los entes encargados de las regulaciones curriculares a nivel estatal. En ellos están predeterminados los temas, los contenidos, los objetivos, los indicadores; en fin, lo que el docente debe hacer en su ejercicio pedagógico. 
- $\quad$ El maestro debe responder, asimismo, a la pregunta ¿a quién enseña? La respuesta o las respuestas que dé a esta pregunta lo ubican en el terreno de diversas disciplinas; por ejemplo, si responde que a quien tiene al frente en el acto de enseñar es un niño, una persona, un ser humano..., estas respuestas bien pueden ubicarlo en un campo teórico determinado, desde el cual la psicología, la sociología, la neurobiología, la política, la administración, la filosofía, la moral, la ética, la biología, la economía, el derecho, etc., le aportan innumerables insumos al momento de abordar los procesos de enseñanza, dado que le permitirán comprender algunas condiciones de desarrollo físico, psicológico, cognitivo, moral, social, afectivo, entre otras, de sus estudiantes. Esto también podría permitirle reconocer las circunstancias y los contextos específicos en los cuales se desarrolla su labor docente, pero también los que circundan la vida misma de sus estudiantes. Todos estos discursos o formas de percibir la realidad le aportan insumos valiosísimos que determinarán en mayor o menor medida sus prácticas pedagógicas. No es lo mismo comprender que se tiene un sujeto al frente, que concebir al alumno como una cifra más dentro del complejo engranaje del sistema educativo.

- Hay otra pregunta ineludible para el maestroy para la pedagogía en general, ¿para qué se enseña? Esta cuestión remite a las intencionalidades y a las teleologías que orientan la acción de enseñar. Las posibles respuestas dejan ver los fines a los que se orienta la educación, los fines que de ella esperan la sociedad, el estado, la cultura, la institución, la familia, el sujeto mismo; una cosa será enseñar con el propósito de lograr determinados objetivos, el desarrollo de determinadas habilidades o competencias; otra cosa será poner la enseñanza al servicio de una capacitación para que responda posteriormente a los procesos económicos y productivos (capacitar mano de obra, por ejemplo); otra cosa significará enseñar para la vida, para que cada sujeto sea dueño de su propio ser, para que sea autónomo, responsable y para que aporte lo mejor de sí (más allá de su fuerza laboral y productiva) a la sociedad. ${ }^{8}$

- ¿ ¿Cómo enseñar? Esta cuestión remite a los modos específicos en los cuales el maestro concreta su labor de enseñar. Una mente abierta a echar mano de la multiplicidad de herramientas que el conocimiento mismo, los desarrollos científico-tecnológicos..., ponen a disposición de la acción de enseñar, tendrá más posibilidades de éxito al enfrentarse a sus alumnos en el aula de clase; ello supone el conocimiento del contexto y el aprovechamiento de las herramientas (didácticas, dinámicas, metodologías, estrategias, etc.) de que puede disponer para el desempeño de su labor. El conocimiento de modelos y teorías de enseñanza-aprendizaje, de metodologías, didácticas y mediaciones pedagógicas, con toda seguridad convertirá su aula de clase en un escenario de aprendizaje con sentido, para la vida.

- ¿ ¿Dónde y cuándo enseñar? Las instituciones y la sociedad misma determinan el lugar y el tiempo donde el maestro desempeña su actividad docente. El nivel educativo en que se halle, el curso que oriente, las tareas que desempeñe; su propio nivel de formación, etc., constituyen condiciones de posibilidad para el ejercicio de la docencia en un tiempo, en un espacio.

Este conjunto plural de preguntas y respuestas, ineludibles para la acción docente y el saber pedagógico, nos permiten colegir que el maestro es, en efecto, el sujeto depositario del saber pedagógico.

8 Recordemos la fábula de los tres canteros. La forma como se percibe una labor determinada, una tarea, un oficio, una actividad, determina los resultados que se obtengan; determina, asimismo, una visión de mundo, de conocimiento, de realidad, una visión de hombre. En estas visiones yacen presentes las intencionalidades. Las tres son opciones válidas, pero según las intencionalidades unas más valiosas que otras (Descalzo, 1995). 
Ahora bien, más allá de esta realidad, podrían establecerse otras posibilidades en torno al rol que el docente pudiera plantear en la sociedad. Al respecto, si bien la noción de campo educativo ${ }^{9}$ planteada por Díaz (1993) alude a la existencia de una élite intelectual que se encarga de producir el conocimiento y sus lineamientos de aplicación en educación y pedagogía, postula también un campo pedagógico, que se encarga de reproducirlo y en el cual están representados los profesores.

La división social del trabajo de control simbólico ha producido diferentes subcampos, entre los cuales, el Campo Intelectual de la Educación es uno de ellos. Este campo se refiere a las posiciones, oposiciones y prácticas que surgen de la producción discursiva y no de la reproducción del discurso educativo y sus prácticas que se realizan en el campo de reproducción al cual hemos denominado campo pedagógico (p.16).

Sin embargo, a nuestro modo de ver, y aunque se esperan nuevos avances al respecto, históricamente se observa un fuerte cambio cualitativo en ese sentido, en la medida que se ha producido un mayor empoderamiento de los profesores en términos de sus análisis, participación y, en general, un carácter propositivo y proactivo frente a las instancias que encargadas de instalar las políticas educativas en la cultura académica. Esto seguramente se asocia al mayor nivel de formación investigativa y académica a la cual están accediendo los profesores. A este respecto, podría señalarse que a más elevado maestro más elevado saber y que a más elevado (amplio) saber, mayores condiciones de posibilidad para que cree, recree, disponga y ponga en funcionamiento diversas estrategias didácticas, dinámicas, metodologías, mediaciones, que favorezcan los procesos de aprendizaje en el aula de clase; ello sin olvidar

9 A partir de la noción de campo planteada por Bourdieu y Foucault, dicho trabajo parte de las experiencias particulares del caso colombiano, como lo es el caso del Movimiento Pedagógico y la Expedición Pedagógica. las condiciones de posibilidad (también reales) que pueden obstaculizar o facilitar la labor pedagógica.

Así mismo, es importante plantear que el maestro poseedor de un amplísimo abanico de conocimientos, conocedor de los modelos pedagógicos, de sus dominios específicos de saber disciplinar, de los procedimientos estratégicos, procedimentales, metodológicos, posee un sinnúmero de condiciones y posibilidades de creación y recreación en términos del conocimiento en el aula de clase. Esto deviene necesariamente en la incorporación de manera permanente de nuevas y mejores formas de enseñanza, lo cual, por supuesto, cualificará su acción de enseñar.

A nuestro modo de ver, es importante tener a disposición diferentes y novedosas posibilidades para realizar efectivos procesos de aprendizaje como la música, el cine, la literatura, la poesía, los cuentos, las fábulas, las leyendas, los ejemplos y la experiencia de la vida cotidiana, lo que se ve y se escucha a cada momento, sucesos actuales y de coyuntura, como insumo para la creación de variados y enriquecidos escenarios que coadyuven al ejercicio académico. En palabras de De Tezanos (2007), "recuperar la condición artesanal de la profesión de maestro, reclama de un oficio ejercido en la creatividad" (p. 20).

\section{A modo de conclusión}

El saber pedagógico, propio del maestro, está constituido por un conjunto de fragmentos y recortes de saberes, de disciplinas y de discursos científicos, de prácticas, de relaciones e interacciones que se traman al interior de la acción educativa y que el maestro pone en funcionamiento cotidianamente en su labor de enseñante.

Como sujeto primero y primario del saber pedagógico, el docente es el depositario de un saber, producido socialmente, que crea y recrea de manera constante en su práctica cotidiana: el saber pedagógico. 
ISSN $0123-1294$ | Educ.Educ. Vol. 19. No. 2 | Mayo-Agosto de 2016 | pp. 241-253.

Universidad de La Sabana | Facultad de Educación

Sin embargo, sus aportes, postulaciones críticas y su propio ejercicio investigativo en función de lo que sabe y hace, genera un importante aporte a la sociedad, lo que implica un proceso de empoderamiento en términos de su capacidad de producción académica y participación social.
El acopio y la reflexión del saber pedagógico por parte de los mismos profesores que los producen se constituyen en condición de posibilidad para la creación de escenarios apropiados para el ejercicio pedagógico, en donde a mayor saber, mayores posibilidades de creación y transformación social.

\section{Referencias}

De Tezanos, A. (2007). Oficio de saber. Saber pedagógico: la relación fundante. Educación y Ciudad, (12).

Descalzo, J. (1995). Razones para el amor. Madrid: Sociedad de Educación Atenas.

Díaz, M. (1993). El campo intelectual de la educación en Colombia. Cali: Universidad del Valle. Textos Universitarios.

Echeverry, J. (1998). Campo intelectual de la educación y campo pedagógico. Posibilidades, complementos y diferencias. Educación y Ciudad (4),12-23.

Foucault, M. (1979). Microfísica del poder (2 ed.). Madrid: La Piqueta.

Foucault, M. (2000). Nietzsche, la genealogía, la historia. Valencia: Pretextos.

Foucault, M. (2003). La verdad y las formas jurídicas (2 ed.). Barcelona: Gedisa.

Foucault, M. (2004). El pensamiento del afuera. Valencia: Pre-textos.

Foucault, M. (2006). La arqueología del saber (22 ed.). México: Siglo XXI.

González, H. (2016). Caracterización del saber pedagógico de los profesores del Proyecto Académico de Investigación y Extensión de Pedagodía-PAIEP en la Universidad Distrital Francisco José de Caldas. Bogotá (no publicada).

González, H.y Florián, V. (1999). Nietzsche-Foucault. Bogotá: Universidad Nacional Abierta y a Distancia-UNAD.

Sánchez, T. (2015). La evaluación en Colombia: segunda mitad del siglo XX. Esbozo de una genealogía. Bogotá: Universidad DistritalFrancisco José de Caldas.

Tamayo, A. (2007). Tendencias de la pedagogía en Colombia. Revista Latinoamericana de Estudios Educativos, 65-76. Recuperado de http://latinoamericana.ucaldas.edu.co/downloads/Latinoamericana3-1_5.pdf

Vasco, E. (1990). El saber pedagógico: razón de ser de la pedagogía. En Díaz, M. Y. Pedagogía, discurso y poder. Bogotá: Corprodic. 
Zuluaga, O. (1987). Pedagogía e historia. la historicidad de la pedagogía. La enseñanza, un objeto de saber. Bogotá: Foro Nacional por Colombia.

Zuluaga, O. (2006). Relaciones entre saber pedagógico, práctica pedagógica y memoria activa del saber pedagógico. En Ouiceno, H. et al. Territorios pedagógicos: espacios, saberes y siujetos I (pp. 91-102). Bogotá: Universidad Pedagógica Nacional. 